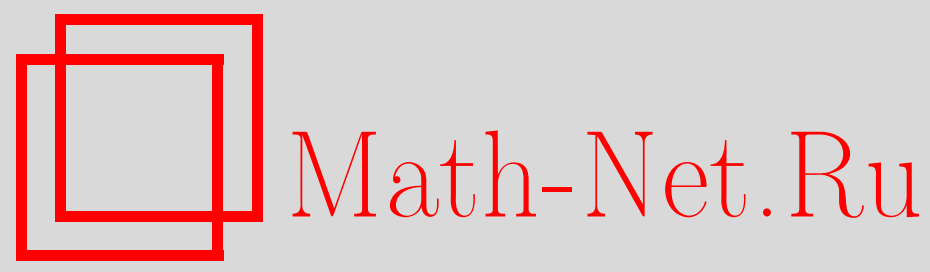

В. А. Едемский, О линейной сложности двоичных последовательностей на основе классов биквадратичных и шестеричных вычетов, Дискрет. матем., 2010, том 22, выпуск $1,74-82$

DOI: https://doi.org/10.4213/dm1085

Использование Общероссийского математического портала Math-Net.Ru подразумевает, что вы прочитали и согласны с пользовательским соглашением http://www . mathnet.ru/rus/agreement

Параметры загрузки:

IP : 52.205 .19 .152

26 апреля 2023 г., 15:50:29 


\title{
О линейной сложности двоичных последовательностей на основе классов биквадратичных и шестеричных вычетов
}

\author{
() 2010 г. В. А. Едемский
}

\begin{abstract}
Предложен метод расчета линейной сложности двоичных периодических последовательностей, сформированных на основе классов биквадратичных и шестеричных вычетов, посредством разложения периода последовательности на сумму квадратов целых чисел. Значения многочлена последовательности вычисляются с использованием циклотомических чисел четвертого и шестого порядков.
\end{abstract}

\section{1. Введение}

Линейная сложность последовательности определяется как длина самого короткого линейного регистра сдвига с обратными связями, с помощью которого можно воссоздать последовательность, и является ее важным показателем [1]. Последовательности, обладающие высокой линейной сложностью, важны для криптографических приложений. Известны алгоритмы определения минимального многочлена последовательности и ее линейной сложности, например, алгоритм Берлекэмпа-Месси [1]. В то же время для ряда периодических последовательностей, сформированных на основе классов степенных вычетов, с хорошими автокорреляционными свойствами линейная сложность определяется видом периода последовательности [2-4].

Цель настоящей статьи заключается в определении линейной сложности двоичных периодических последовательностей, сформированных на основе классов биквадратичных и шестеричных вычетов, посредством разложения периода последовательности на сумму квадратов целых чисел. Значения многочлена последовательности вычисляются с использованием циклотомических чисел четвертого и шестого порядков. Данный метод может быть использован для вычисления линейной сложности других последовательностей на основе классов степенных вычетов при известных циклотомических числах.

Пусть $p=d R+1-$ простое число, где $d, R-$ натуральные числа, $d \neq 1$ и $H_{k}-$ класс степенных вычетов с номером $k$ :

$$
H_{k}=\left\{\theta^{k+d t}, t=0,1, \ldots, R-1\right\}, \quad k=0,1, \ldots, d-1,
$$

где $\theta$ - первообразный корень по простому модулю. 
Рассмотрим двоичную последовательность $X=\left\{x_{i}\right\}$ периода $p$, сформированную на основе классов степенных вычетов:

$$
x_{i}= \begin{cases}1, & i \in H_{k}, k \in I, \\ 0 & \text { в остальных случаях. }\end{cases}
$$

Здесь $I-$ непустое подмножество множества индексов от 0 до $d-1$. Обозначим

$$
S(t)=x_{0}+x_{1} t+\ldots+x_{p-1} t^{p-1}
$$

многочлен последовательности $X$, принадлежащий $G F(2)[t]$. Согласно [2], линейная сложность последовательности $L$ определяется соотношением

$$
L=p-\left|\left\{n \mid S\left(\alpha^{n}\right)=0, n=0,1, \ldots, p-1\right\}\right|,
$$

где $\alpha$ - примитивный корень $p$ степени из единицы в поле разложения $K$ многочлена $t^{p}-1$ над $G F(2)$. А так как $\theta-$ первообразный корень по модулю $p$, задача вычисления линейной сложности последовательности $X$ сводится к определению значений $S\left(\alpha^{\theta^{l}}\right)$, $l=1, \ldots, p-1$, и $S(1)$ в поле $K$.

В следующем разделе рассматривается способ вычисления значений $S\left(\alpha^{\theta^{l}}\right)$.

\section{2. Многочлен последовательности и циклотомические числа}

Введем дополнительный многочлен

$$
S_{d}(t)=\sum_{n \in H_{0}} t^{n}
$$

Следующая лемма является несложным обобщением утверждений, доказанных в [2, 4] для $d=2$ и $d=6$.

Лемма 1. Для $f=0,1, \ldots, d-1$ справедливы равенства

(1) $\sum_{n \in H_{f}} \alpha^{n}=S_{d}\left(\alpha^{\theta^{f}}\right)$ для $f=0,1, \ldots, d-1$;

(2) $S_{d}\left(\alpha^{\theta^{f+d g}}\right)=S_{d}\left(\alpha^{\theta^{f}}\right)$ для любого ичелого числа $g$;

(3) если $\operatorname{ind}_{\theta} 2 \equiv l(\bmod d)$, mо $S_{d}\left(\alpha^{\theta^{l}}\right)=S_{d}^{2}(\alpha)$;

(4) если $\operatorname{ind}_{\theta} 2 \equiv 0(\bmod d)$, то $S_{d}\left(\alpha^{\theta^{g}}\right) \in\{0,1\}$ для любого ичелого числа $g$;

(5) $S_{d}\left(\alpha^{\theta^{f}}\right) \neq 1$ хотя бы для одного $f=0,1, \ldots, d-1$.

Так как

$$
0=\alpha^{p}-1=(\alpha-1)\left(1+\alpha+\ldots+\alpha^{p-1}\right),
$$

согласно лемме 1, в поле характеристики два

$$
S_{d}(\alpha)+S_{d}\left(\alpha^{\theta}\right)+\ldots+S_{d}\left(\alpha^{\theta^{d-1}}\right)=1 .
$$


Согласно определению,

$$
S\left(\alpha^{\theta^{l}}\right)=\sum_{k \in I} S_{d}\left(\alpha^{\theta^{k+l}}\right)
$$

таким образом, в силу леммы 1 и определения (2) для нахождения линейной сложности $L$ достаточно найти значения $S_{d}\left(\alpha^{\theta^{f}}\right), f=0,1, \ldots, d-1$.

Обозначим $(k, f)_{d}$ циклотомические числа порядка $d$ (см. [5]).

Теорема 1. При $k=0,1, \ldots, d-1$

$$
S_{d}(\alpha) S_{d}\left(\alpha^{\theta^{k}}\right)=\sum_{f=0}^{d-1}(k, f)_{d} S_{d}\left(\alpha^{\theta^{f}}\right)+\delta
$$

2de

$$
\delta= \begin{cases}R, & \text { если } R \equiv 0 \quad(\bmod 2), k=0 \text { или } R \equiv 1 \quad(\bmod 2), k=d / 2, \\ 0 & \text { в остальных случаях. }\end{cases}
$$

Доказательство. Согласно определению $S_{d}(t)$ и $H_{0}$,

$$
S_{d}(\alpha) S_{d}\left(\alpha^{\theta^{k}}\right)=\sum_{u, v=0}^{R-1} \alpha^{\theta^{d u}+\theta^{k+d v}}
$$

Если $\theta^{d u}+\theta^{k+d v} \not \equiv 0(\bmod p)$, то существуют числа $f, s, f \in\{0,1, \ldots, d-1\}$, $s=\{0,1, \ldots, R-1\}$, такие, что $\theta^{d u}+\theta^{d v} \equiv \theta^{f+d s}(\bmod p)$. При каждом значении $s$ число пар $(u, v)$, являющихся решениями последнего сравнения, совпадает с циклотомическим числом $(k, f)_{d}$ порядка $d$ (см. [5]). Таким образом,

$$
\sum_{u, v=0}^{R-1} \alpha^{\theta^{d u}+\theta^{k+d v}}=\sum_{f=0}^{d-1} \sum_{s=0}^{R-1}(k, f)_{d} \alpha^{\theta^{f+d s}}+\delta=\sum_{f=0}^{d-1}(k, f)_{d} S_{d}\left(\alpha^{\theta^{f}}\right)+\delta,
$$

где $\delta$ равно числу решений сравнения $\theta^{d u}+\theta^{k+d v} \equiv 0(\bmod p)$. Справедливо сравнение $-1 \equiv \theta^{d R / 2}(\bmod p)$, поэтому число $\delta$ именно таково, как указано в условиях теоремы, что и требовалось доказать.

Следствие 1. Для любого цчелого $l$

$$
S_{d}\left(\alpha^{\theta^{l}}\right) S_{d}\left(\alpha^{\theta^{k+l}}\right)=\sum_{f=0}^{d-1}(k-l, f)_{d} S_{d}\left(\alpha^{\theta^{f}}\right)+\delta .
$$

Теорема 1 и следствие 1 позволяют рассчитать значения $S_{d}\left(\alpha^{\theta^{f}}\right), f=0,1, \ldots, d-1$, а значит и $S\left(\alpha^{\theta^{f}}\right), f=0,1, \ldots, d-1$, при известных циклотомических числах порядка $d$, что, согласно (2), делает возможным определение линейной сложности последовательности.

Воспользовавшись известными формулами для вычисления циклотомических чисел $[5,6]$, в следующих разделах мы определим линейную сложность последовательностей (1) для $d=4,6$. 


\section{3. Линейная сложность последовательностей на основе классов биквадратичных вычетов с периодом $p=4 R+1$}

Циклотомические числа четвертого порядка определяются разложением (см. [5])

$$
p=x^{2}+4 y^{2}, \quad x=1+4 z,
$$

где $x, y, z$ - целые числа, и зависят от четности $R$, совпадающей с четностью $y$.

3.1. Пусть $R \equiv 0(\bmod 2)$, тогда $y=2 w$. Рассчитав по формулам, приведенным в [5], вычеты циклотомических чисел четвертого порядка по модулю два

$$
\begin{aligned}
& 16(2,0)_{4}=16(2,2)_{4}=p-3+2 x, \\
& 16(2,1)_{4}=16(2,3)_{4}=p+1-2 x,
\end{aligned}
$$

получаем по теореме 1 и следствию 1 в поле $K$ следующую систему:

$$
\begin{aligned}
S_{4}(\alpha) S_{4}\left(\alpha^{\theta^{2}}\right) & =w\left(S_{4}(\alpha)+S_{4}\left(\alpha^{\theta^{2}}\right)\right)+(z+w)\left(S_{4}\left(\alpha^{\theta}\right)+S_{4}\left(\alpha^{\theta^{3}}\right)\right), \\
S_{4}\left(\alpha^{\theta}\right) S_{4}\left(\alpha^{\theta^{3}}\right) & =(z+w)\left(S_{4}(\alpha)+S_{4}\left(\alpha^{\theta^{2}}\right)\right)+w\left(S_{4}\left(\alpha^{\theta}\right)+S_{4}\left(\alpha^{\theta^{3}}\right)\right) .
\end{aligned}
$$

Если $y \equiv 0(\bmod 2)$, то $\operatorname{ind}_{\theta} 2 \equiv 0(\bmod 2)($ см. [7]). Согласно лемме 1 и равенству (3), можно считать, не нарушая общности, что $S_{2}(\alpha)=1, S_{2}\left(\alpha^{\theta}\right)=0$. Тогда

$$
S_{4}(\alpha)+S_{4}\left(\alpha^{\theta^{2}}\right)=1, \quad S_{4}\left(\alpha^{\theta}\right)+S_{4}\left(\alpha^{\theta^{3}}\right)=0,
$$

и система (4) примет вид

$$
\begin{aligned}
& S_{4}(\alpha) S_{4}\left(\alpha^{\theta^{2}}\right),=z \\
& S_{4}\left(\alpha^{\theta}\right) S_{4}\left(\alpha^{\theta^{3}}\right)=z+w .
\end{aligned}
$$

Пусть $\mathbf{S}=\left(S_{4}(\alpha), S_{4}\left(\alpha^{\theta}\right), S_{4}\left(\alpha^{\theta^{2}}\right), S_{4}\left(\alpha^{\theta^{3}}\right)\right)$. Решая систему (5), получаем, что

(1) $\mathbf{S}=(1,0,0,0)$ или $S=(0,0,1,0)$, если $z \equiv 0(\bmod 2), w \equiv 0(\bmod 2)$;

(2) $\mathbf{S}=(1,1,0,1)$ или $S=(0,1,1,1)$, если $z \equiv 1(\bmod 2), w \equiv 0(\bmod 2)$;

(3) $\mathbf{S}=(\gamma, 1,1-\gamma, 1)$, где $\gamma-$ корень уравнения $\gamma^{2}+\gamma+1=0$, если $z \equiv 0(\bmod 2)$, $w \equiv 1(\bmod 2)$

(4) $\mathbf{S}=(\gamma, 0,1-\gamma, 0)$, если $z \equiv 1(\bmod 2), w \equiv 1(\bmod 2)$.

Найденные значения $S_{4}(\alpha), S_{4}\left(\alpha^{\theta}\right), S_{4}\left(\alpha^{\theta^{2}}\right), S_{4}\left(\alpha^{\theta^{3}}\right)$ позволяют определить по (2) линейную сложность любой последовательности, сформированной по (1) на основе классов биквадратичных вычетов. В частности, имеет место следующее утверждение.

Лемма 2. Если

$$
p=(1+4 z)^{2}+16 w^{2}
$$


и двоичная последовательность сформирована по (1) на основе одного класса биквадратичных вычетов, то ее линейная сложность

$$
L_{1}=\left\{\begin{array}{llll}
(p-1) / 4, & z \equiv 0 & (\bmod 2), w \equiv 0 & (\bmod 2), \\
3(p-1) / 4, & z \equiv 1 & (\bmod 2), w \equiv 0 & (\bmod 2), \\
p-1, & z \equiv 0 & (\bmod 2), w \equiv 1 & (\bmod 2), \\
(p-1) / 2, & z \equiv 1 & (\bmod 2), w \equiv 1 & (\bmod 2),
\end{array}\right.
$$

а если на основе трех классов биквадратичных вычетов, то

$$
L_{3}=\left\{\begin{array}{llll}
3(p-1) / 4, & z \equiv 0 & (\bmod 2), w \equiv 0 & (\bmod 2), \\
(p-1) / 4, & z \equiv 1 & (\bmod 2), w \equiv 0 & (\bmod 2), \\
(p-1) / 2, & z \equiv 0 & (\bmod 2), w \equiv 1 & (\bmod 2), \\
p-1, & z \equiv 1 & (\bmod 2), w \equiv 1 & (\bmod 2)
\end{array}\right.
$$

Доказательство. Если последовательность сформирована на основе одного класса биквадратичных вычетов, то число $f=0,1,2,3$ таких, что $S\left(\alpha^{\theta^{f}}\right)=0$ совпадает с числом нулей в матрице $\mathbf{S}$, а если же $X$ сформирована на основе трех классов, то с числом единиц в матрице $\mathbf{S}$, так как $S\left(\alpha^{\theta^{f}}\right)=1-S_{4}\left(\alpha^{\theta^{f}}\right)$ согласно (3). Применение формулы (2) с учетом леммы 1 завершает доказательство.

Если последовательность сформирована на основе двух классов биквадратичных вычетов, то при $I=\{0,1\}$ ее линейная сложность

$$
L_{2}=\left\{\begin{array}{lll}
(p-1) / 2, & \text { если } w \equiv 0 & (\bmod 2), \\
p-1, & \text { если } w \equiv 1 & (\bmod 2),
\end{array}\right.
$$

а при $I=\{0,2\}$ значение $L_{2}=(p-1) / 2$ для $p=(1+4 z)^{2}+16 w^{2}$. Это известный результат $[2,3]$.

\section{2. Пусть $R \equiv 1(\bmod 2)$.}

Лемма 3. Если

$$
p=x^{2}+4(1+2 w)^{2}
$$

и двоичная последовательность сформирована по (1), то при любом числе классов биквадратичных вычетов, отличном от четырех, ее линейная сложность

$$
L=\left\{\begin{array}{lll}
p, & \text { если } R|I| \equiv 1 & (\bmod 2), \\
p-1, & \text { если } R|I| \equiv 0 & (\bmod 2) .
\end{array}\right.
$$

Доказательство. Если $y \equiv 1(\bmod 2)$, то $\operatorname{ind}_{\theta} 2 \not \equiv 0(\bmod 2)($ см. [7]). Не нарушая общности, будем считать, что $\operatorname{ind}_{\theta} 2 \equiv 1(\bmod 4)\left(\right.$ если $\operatorname{ind}_{\theta} 3 \equiv 3(\bmod 4)$, то заменим $\theta$ на $\left.\theta^{-1}\right)$. Тогда, в силу леммы $1, S_{4}\left(\alpha^{\theta}\right)=S_{4}^{2}(\alpha)$, следовательно, и $S\left(\alpha^{\theta}\right)=S^{2}(\alpha)$. Таким образом, либо все значения $S\left(\alpha^{\theta^{l}}\right)=0$, либо $S\left(\alpha^{\theta^{l}}\right) \neq 0$ при $l=1, \ldots, p-1$. Первый вариант невозможен, если число классов биквадратичных вычетов, используемых при построении последовательности, меньше четырех.

Таким образом, линейная сложность последовательности $L=p-1$, если $S(1)=0$, и $L=p$ в противном случае. 
Таблица 1. Вычеты циклотомических чисел шестого порядка по модулю два

\begin{tabular}{|c|c|c|c|}
\hline & \multicolumn{3}{|c|}{$R \equiv 1(\bmod 2), A=4+6 a$} \\
\hline $\bar{B}$ & $3+6 b$ & $5+6 b$ & $1+6 b$ \\
\hline$(0,0)_{6}$ & $\bar{a}$ & 0 & 0 \\
\hline$(0,1)_{6}$ & 0 & $1+a$ & 0 \\
\hline$(0,2)_{6}$ & 0 & 0 & $a$ \\
\hline$(0,3)_{6}$ & $1+a$ & 0 & 0 \\
\hline$(0,4)_{6}$ & 0 & $a$ & 0 \\
\hline$(0,5)_{6}$ & 0 & 0 & $1+a$ \\
\hline$(1,0)_{6}$ & $a+b$ & $1+b$ & $1+a+b$ \\
\hline$(2,0)_{6}$ & $1+a+b$ & $a+b$ & $b$ \\
\hline$(1,2)_{6}$ & 1 & $1+a$ & $1+a$ \\
\hline$(2,1)_{6}$ & 1 & $a$ & $a$ \\
\hline
\end{tabular}

\begin{tabular}{|c|c|c|c|}
\hline & \multicolumn{3}{|c|}{$R \equiv 0(\bmod 2), A=1+6 a$} \\
\hline$B$ & $6 b$ & $2+6 b$ & $4+6 b$ \\
\hline$(0,0)_{6}$ & $1+b$ & 0 & 0 \\
\hline$(0,1)_{6}$ & 0 & $1+b$ & 0 \\
\hline$(0,2)_{6}$ & 0 & 0 & $1+b$ \\
\hline$(0,3)_{6}$ & $b$ & 0 & 0 \\
\hline$(0,4)_{6}$ & 0 & $b$ & 0 \\
\hline$(0,5)_{6}$ & 0 & 0 & $b$ \\
\hline$(1,2)_{6}$ & $a$ & $a$ & $a$ \\
\hline$(1,3)_{6}$ & $a$ & $a$ & $a+b$ \\
\hline$(1,4)_{6}$ & $a$ & $1+a+b$ & $a$ \\
\hline$(2,4)_{6}$ & $a$ & $1+a$ & $1+a$ \\
\hline
\end{tabular}

Наибольший общий делитель

$$
\operatorname{GCD}\left(t^{p}-1, S(t)\right)= \begin{cases}\prod_{f} \prod_{s=0}^{R-1}\left(t-\alpha^{\theta^{f+4 s}}\right), & S\left(\alpha^{\theta^{f}}\right)=0, S(1) \neq 0, \\ (t-1) \prod_{f} \prod_{s=0}^{R-1}\left(t-\alpha^{\theta^{f+4 s}}\right), & S\left(\alpha^{\theta^{f}}\right)=0, S(1)=0,\end{cases}
$$

поэтому найденные значения $S_{4}\left(\alpha^{\theta^{l}}\right)$ позволяют также найти характеристический многочлен последовательности $f(t)=\left(t^{p}-1\right) / \operatorname{GCD}\left(t^{p}-1, S(t)\right)$.

\section{4. Линейная сложность последовательностей на основе классов шестеричных вычетов с периодом $p=6 R+1$}

Циклотомические числа шестого порядка определяются разложением $p=A^{2}+3 B^{2}$, $A \equiv 1(\bmod 3)($ см. $[5,6])$, которое определяет значение $A$ однозначно, $B-$ с точностью до знака. Знак $B$ определим, как в [6], при этом, если $B \equiv 0(\bmod 3)$, то $B / 3 \equiv \operatorname{ind}_{\theta} 3$ $(\bmod 3)$ и всегда $B \equiv-\operatorname{ind}_{\theta} 2(\bmod 3)$. Если $A \equiv 1(\bmod 3)$, то четность $R$ совпадает с четностью $B$. Воспользовавшись формулами для циклотомических чисел шестого порядка из $[5,6]$, приведем табл. 1 для вычетов циклотомических чисел по модулю два $(a, b-$ целые числа).

В следующих двух подраздела, используя теорему 1 , вычислим значения $S_{6}\left(\alpha^{\theta^{f}}\right)$.

4.1. Пусть $B \equiv 0(\bmod 3)$, то есть $\operatorname{ind}_{\theta} 2 \equiv 0(\bmod 3)$.

Согласно (3) и лемме 1 одно из чисел $S_{3}(\alpha), S_{3}\left(\alpha^{\theta}\right), S_{3}\left(\alpha^{\theta^{2}}\right)$ равно единице, а остальные - нулю, не нарушая общности, можно считать, что $S_{3}(\alpha)=1$. Тогда

$$
\begin{aligned}
S_{6}(\alpha)+S_{6}\left(\alpha^{\theta^{3}}\right) & =1, \\
S_{6}\left(\alpha^{\theta}\right)+S_{6}\left(\alpha^{\theta^{4}}\right) & =0, \\
S_{6}\left(\alpha^{\theta^{2}}\right)+S_{6}\left(\alpha^{\theta^{5}}\right) & =0 .
\end{aligned}
$$


Воспользовавшись теоремой 1, табл. 1, равенством (6) и свойствами циклотомических чисел, получаем следующие системы уравнений: если $R \equiv 1(\bmod 2)$, то

$$
\begin{aligned}
S_{6}(\alpha) S_{6}\left(\alpha^{\theta^{3}}\right) & =a+1, \\
S_{6}\left(\alpha^{\theta}\right) S_{6}\left(\alpha^{\theta^{4}}\right) & =a+b, \\
S_{6}\left(\alpha^{\theta^{2}}\right) S_{6}\left(\alpha^{\theta^{5}}\right) & =a+b+1 ;
\end{aligned}
$$

если $R \equiv 0(\bmod 2)$, то

$$
\begin{aligned}
S_{6}(\alpha) S_{6}\left(\alpha^{\theta^{3}}\right) & =b, \\
S_{6}\left(\alpha^{\theta}\right) S_{6}\left(\alpha^{\theta^{4}}\right) & =a, \\
S_{6}\left(\alpha^{\theta^{2}}\right) S_{6}\left(\alpha^{\theta^{5}}\right) & =a .
\end{aligned}
$$

Дополнительно отметим, что если $p \equiv \pm 1(\bmod 8), a \equiv 1(\bmod 2)$ при нечетном $R$ или $b \equiv 0(\bmod 2)$ при четном $R$, то $\operatorname{ind}_{\theta} 2 \equiv 0(\bmod 6)($ см. [7]) и можно считать, что $S_{6}(\alpha)=1$, в силу леммы 1 и (6).

Решая системы (7) и (8), с учетом (6) и сделанного замечания, получим следующие варианты для $\mathbf{S}=\left(S_{6}(\alpha), S_{6}\left(\alpha^{\theta}\right), \ldots, S_{6}\left(\alpha^{\theta^{5}}\right)\right)$ : если $R \equiv 1(\bmod 2)$, то

(1) $\mathbf{S}=(1,1,0,0,1,0)$, если $a \equiv 1(\bmod 2), b \equiv 0(\bmod 2)$;

(2) $\mathbf{S}=(1,0,1,0,0,1)$, если $a \equiv 1(\bmod 2), b \equiv 1(\bmod 2)$;

(3) $\mathbf{S}=(\gamma, 0,1,1-\gamma, 0,1)$, где $\gamma-$ корень уравнения $\gamma^{2}+\gamma+1=0$, если $a \equiv 0$ $(\bmod 2), b \equiv 0(\bmod 2)$;

(4) $\mathbf{S}=(\gamma, 1,0,1-\gamma, 1,0)$, если $a \equiv 0(\bmod 2), b \equiv 1(\bmod 2)$;

если же $R \equiv 0(\bmod 2)$, то

(1) $\mathbf{S}=(1,0,0,0,0,0)$, если $a \equiv 0(\bmod 2), b \equiv 0(\bmod 2)$;

(2) $\mathbf{S}=(1,1,1,0,1,1)$, если $a \equiv 1(\bmod 2), b \equiv 0(\bmod 2)$;

(3) $\mathbf{S}=(\gamma, 0,0,1-\gamma, 0,0)$, если $a \equiv 0(\bmod 2), b \equiv 1(\bmod 2)$;

(4) $\mathbf{S}=(\gamma, 1,1,1-\gamma, 1,1)$, если $a \equiv 1(\bmod 2), b \equiv 1(\bmod 2)$.

Найденные значения $S_{6}\left(\alpha^{\theta^{f}}\right), f=0,1, \ldots, 5$ позволяют рассчитать линейную сложность любой двоичной последовательности $X$, построенной по (1) на основе классов шестеричных вычетов в зависимости от разложения $p=A^{2}+3 B^{2}$, где $B \equiv 0(\bmod 3)$. Здесь ограничимся нахождением линейной сложности почти уравновешенных последовательностей при нечетном $R$. В связи с тем, что линейная сложность последовательности $X$, построенной по (1), не меняется при циклическом сдвиге индексов из множества $I$, достаточно рассмотреть только два варианта: $I=\{0,1,2\}, I=\{0,1,3\}$ (см. [8]).

Согласно (2), линейная сложность последовательности определяется числом значений $l$ таких, что $S\left(\alpha^{\theta^{l}}\right)=0$. Так как

$$
S\left(\alpha^{\theta^{l}}\right)=\sum_{k \in I} S_{d}\left(\alpha^{\theta^{k+l}}\right),
$$

значения многочлена последовательности определяются суммированием элементов матрицы S. Воспользовавшись найденными значениями элементов матрицы $\mathbf{S}$, получаем следующее утверждение. 
Лемма 4. Если

$$
p=4(2+3 a)^{2}+27(1+2 b)^{2}
$$

и двоичная последовательность сформирована по (1) для $I=\{0,1,2\}$, то ее линейная сложность L задается равенствами

$$
L=\left\{\begin{array}{lll}
(p-1) / 2+1, & \text { если } a \equiv 1 & (\bmod 2) \\
p, & \text { если } a \equiv 0 & (\bmod 2),
\end{array}\right.
$$

а при $I=\{0,1,3\}$

$$
L=\left\{\begin{array}{llll}
(p-1) / 6+1, & \text { если } a \equiv 1 & (\bmod 2), b \equiv 0 & (\bmod 2), \\
(p-1) / 3+1, & \text { если } a \equiv 0 & (\bmod 2), b \equiv 1 & (\bmod 2), \\
5(p-1) / 6+1, & \text { если } a \equiv 1 & (\bmod 2), b \equiv 1 & (\bmod 2), \\
p, & \text { если } a \equiv 0 & (\bmod 2), b \equiv 0 & (\bmod 2)
\end{array}\right.
$$

В частном случае, когда $b=0(B=3)$, линейная сложность последовательности Холла определена в [4]. Метод, используемый в [4], не может быть использован для $B \neq 3$.

Линейная сложность других двоичных последовательностей рассчитывается подобным же образом. Характеристический многочлен последовательности определяется, как и при $d=4$.

4.2. Пусть $B \not \equiv 0(\bmod 3)$.

Пусть $B \equiv 1(\bmod 3)$ для нечетного $R$ и $B \equiv 4(\bmod 3)$ для четного $R$, тогда $\operatorname{ind}_{\theta} 2 \equiv 2(\bmod 3)$.

Если $\operatorname{ind}_{\theta} 2 \equiv 5(\bmod 6)$, то, как и в разделе 3.2 , получаем, что

$$
L=\left\{\begin{array}{lll}
p, & \text { если } R|I| \equiv 1 & (\bmod 2), \\
p-1, & \text { если } R|I| \equiv 0 & (\bmod 2),
\end{array}\right.
$$

при любом числе классов степенных вычетов, отличном от шести.

Пусть $\operatorname{ind}_{\theta} 2 \equiv 2(\bmod 6)$, тогда $p \equiv \pm 1(\bmod 8)($ см. [7]), и по лемме 1

$$
S_{6}\left(\alpha^{\theta^{2}}\right)=S_{6}^{2}(\alpha)
$$

и, как и в разделе 3.1 , можно считать, не нарушая общности, что $S_{2}(\alpha)=1, S_{2}\left(\alpha^{\theta}\right)=0$, то есть

$$
S_{6}(\alpha)+S_{6}^{2}(\alpha)+S_{6}^{4}(\alpha)=1 .
$$

Если $R-$ нечетное число, то $a \equiv 1(\bmod 2)$ и по теореме 1

$$
S_{6}(\alpha) S_{6}\left(\alpha^{\theta^{2}}\right)=b S_{6}(\alpha)+S_{6}\left(\alpha^{\theta}\right)+b S_{6}\left(\alpha^{\theta^{2}}\right) .
$$

Согласно (9), (11) и лемме 1 , равенство $S_{6}(\alpha)=1$ невозможно, то есть $S_{6}(\alpha)=\lambda$, где $\lambda$ - корень уравнения $\lambda^{3}+\lambda^{2}+1=0$ согласно (10). Из (11) получаем, что

(1) $\mathbf{S}=\left(\lambda, \lambda^{2}+1, \lambda^{2}, \lambda^{2}+\lambda, \lambda^{2}+\lambda+1, \lambda+1\right)$, если $R \equiv 1(\bmod 2), a \equiv 1(\bmod 2)$, $b \equiv 0(\bmod 2)$ 
(2) $\mathbf{S}=\left(\lambda, \lambda+1, \lambda^{2}, \lambda^{2}+1, \lambda^{2}+\lambda+1, \lambda^{2}+\lambda\right)$, если $R \equiv 1(\bmod 2), a \equiv 1(\bmod 2)$, $b \equiv 1(\bmod 2)$.

Если же $R-$ четное число, то $b \equiv 0(\bmod 2)$, и по теореме 1

$$
S_{6}(\alpha) S_{6}\left(\alpha^{\theta}\right)=a\left(S_{6}\left(\alpha^{\theta^{2}}\right)+S_{6}\left(\alpha^{\theta^{3}}\right)+S_{6}\left(\alpha^{\theta^{4}}\right)+S_{6}\left(\alpha^{\theta^{5}}\right)\right) .
$$

Решая систему (12), получаем, что

(1) $\mathbf{S}=\left(\lambda, 0, \lambda^{2}, 0, \lambda^{2}+\lambda+1,0\right)$, если $R \equiv 0(\bmod 2), a \equiv 0(\bmod 2), b \equiv 0(\bmod 2)$;

(2) $\mathbf{S}=\left(1, \beta, 1, \beta^{2}, 1, \beta^{2}+\beta\right)$, если $R \equiv 0(\bmod 2), a \equiv 1(\bmod 2), b \equiv 0(\bmod 2)$, где $\beta$ - корень уравнения $\beta^{3}+\beta+1=0$.

Вариант, когда $B \equiv 2(\bmod 3)$, сводится к уже рассмотренному случаю заменой $\theta$ на $\theta^{-1}$.

Таким образом, вычисленные значения $S_{6}\left(\alpha^{\theta^{f}}\right), f=0,1, \ldots, 5$, позволяют рассчитать линейную сложность любых двоичных последовательностей, сформированных по (1) на основе классов шестеричных вычетов, в зависимости от разложения $p=A^{2}+3 B^{2}$ и при $B \not \equiv 0(\bmod 3)$.

Предложенный метод может быть использован для определения линейной сложности и для других последовательностей на основе классов степенных вычетов при известных циклотомических числах.

\section{Список литературы}

1. Лидл Р., Нидеррайтер Г., Конечные поля. Мир, Москва, 1988.

2. Ding C., Helleseth T., Shan W., On the linear complexity of Legendre sequences. IEEE Trans. Inf. Theory (1998) 44, №3, 1275-1278.

3. Ding C., Helleseth T., Lam K. Y., Several classes of binary sequences with tree-level autocorrelation. IEEE Trans. Inf. Theory (1999) 45, 2601-2606.

4. Kim J. H., Song H. Y., On the linear complexity of Hall's sextic residue sequences. IEEE Trans. Inf. Theory (2001) 47, 2094-2096.

5. Hall M., Combinatorial theory. Blaisdell, London, 1967.

6. Whiteman A. L., The cyclotomic numbers of order twelve. Acta Arithmetica (1960) 6, 53-76.

7. Ireland K., Rosen M., A classical introduction to modern number theory. Springer, Berlin, 1982.

8. Гантмахер В. Е., Едемский В. А., Результаты синтеза двоичных последовательностей с квазиодноуровневой автокорреляционной функцией, формируемых на основе классов вычетов по простому модулю. Известия вузов. Радиоэлектроника (2007) №4, 14-23.

Статья поступила 28.04.2008. 